\title{
ESTUDO DO RISCO NA CONCESSÃO DE OUTORGA PARA LANÇAMENTOS DIFUSOS DE EFLUENTES EM RIOS UTILIZANDO UMA METODOLOGIA FUZZY
}

\author{
Sílvia Helena Lima dos Santos ${ }^{1}$ \\ Raquel Jucá de Moraes Sales ${ }^{2}$ \\ Patrícia Freire Chagas ${ }^{3}$ \\ Vanessa Ueta ${ }^{4}$
}

Raimundo Oliveira de Souza ${ }^{5}$ 
RESUMO: Este trabalho trata do desenvolvimento de uma metodologia que combina a teoria fuzzy com a teoria do transporte de massa, para determinar o risco na concessão de outorga de lançamentos de efluentes. O modelo consiste de resolver a Equação da Difusão Advectiva, em uma dimensão, na sua forma fuzzy. Deste modo, campos de funções de pertinências para a concentração são determinados em cada seção do rio, para diferentes tempos. Estas concentrações, em forma de funções de pertinências são comparadas com uma função de pertinência para a resistência do rio. Esta resistência é, normalmente, determinada por normas técnicas, em função da classe de cada corpo hídrico. Assim, uma função marginal de segurança é definida, que permitirá a determinação dos campos de risco ao longo do rio em estudo. Os resultados mostram que esta metodologia pode ser uma alternativa consistente no controle de concessão de outorga de lançamentos e com isso disponibilizando informações para a melhor Gestão dos Recursos Hídricos.

Palavras-chave: Teoria Fuzzy. Concessão de Outorga.Transporte de Massa.

\section{INTRODUÇÃO}

Para disciplinar os usos dos corpos aquáticos, inclusive no que diz respeito à assimilação de efluentes, surgiram leis especificamente para recursos hídricos. Neste contexto, a Política Nacional de Recursos Hídricos, instituída no Brasil pela Lei Federal 9.433, de 08 de janeiro de 1997, é uma importante ferramenta legal para o gerenciamento da água, pela qual é sugerida a utilização de modelos matemáticos e computacionais no suporte à decisão entre alternativas de gestão ou de uso dos recursos hídricos.

De acordo com esta lei um dos principais instrumentos de gestão para redução e controle da poluição desses recursos é a outorga para diluição de efluentes que, apesar de estar legalmente instituída, ainda não foi devidamente implantada em nível nacional. Para garantir que isto ocorra é necessário definir critérios de outorga, organizar e manter uma base de dados de qualidade da água bem como desenvolver ferramentas adequadas 
para uma análise integrada dos aspectos de quantidade e qualidade da água (NAHON, 2006).

A modelagem de um processo físico, presente em um sistema hídrico qualquer não se constitui uma tarefa simples tendo em vista que em corpos d'água naturais ocorrem fenômenos de transferência de massa, energia e quantidade de movimento, que fazem com que estas concentrações não sejam uniformes e dependam fortemente da hidrodinâmica destes corpos d'água. Outro fator que deve ser levado em conta na modelagem destes processos são as incertezas que podem estar relacionadas com os dados, com as medições dos parâmetros, com os métodos de análises e até mesmo com as aproximações das soluções. Desta forma, a análise de incertezas tem um papel fundamental na gestão de Recursos Hídricos e a técnica apropriada para lidar com o problema constitui-se nos fundamentos da análise de risco.

Uma metodologia que esta começando a ser usada nos estudos das incertezas e na Análise de Risco é a Teoria Fuzzy. Esta teoria, desenvolvida nos anos 60, vem se tornando uma ferramenta útil para a análise desta classe de problema, por não depender de um banco de dados tão completo. A grande dificuldade, com relação à aplicação da Teoria Fuzzy nos problemas ambientais reside no fato de que as Equações Diferenciais que governam os processos de transporte da massa poluente precisam ser "fuzzificadas". Isto quer dizer, em outras palavras, que essas equações diferenciais têm que ser transformadas em novas equações diferenciais com características "fuzzy".

Desta forma, este estudo desenvolveu uma metodologia que combinou a Teoria Fuzzy com os Processos de Transporte de Poluentes em um rio natural, e a Legislação Brasileira, para estudar o Risco Fuzzy de contaminação de rios naturais, na concessão de outorga para lançamentos de efluentes.

\section{OUTORGA PARA LANÇAMENTO DE EFLUENES}

A Lei 9.433, de 8.1.1997, define, em seu Art. 12, inciso III, que o lançamento em corpo de água de esgotos e demais resíduos líquidos ou gasosos, tratados ou não, com o fim de sua diluição, transporte ou disposição final, como um uso da água estão sujeitos a 
outorga. Embora constitua um dos principais instrumentos para redução e controle da poluição de recursos hídricos, a outorga para diluição de efluentes em corpos de água, apesar de legalmente instituída, apresenta inúmeras dificuldades que podem retardar sua regulamentação. Segundo Azevedo et al (2003), a emissão da outorga para este fim está atrelada, entre outros fatores, à definição de critérios de que subsidiem a tomada de decisão e ao desenvolvimento de ferramentas adequadas para análise integrada dos aspectos de quantidade e qualidade da água, conforme preconizado no artigo $3^{\circ}$ da Lei Federal 9.433/97, que estabelece a Política Nacional de Recursos Hídricos. Cruz (2001) enfatiza, ainda, a necessidade do estabelecimento de tais critérios, com vistas a otimizar a distribuição da água disponível pelo poder outorgante, sem desconsiderar as particularidades locais e regionais.

A outorga para diluição de efluentes baseia-se no princípio de permitir uma descarga em um curso de água com uma carga máxima de poluentes de maneira que, após sua diluição na vazão mínima fixada como referência, a qualidade da água no corpo receptor permaneça satisfatória, conforme seus objetivos de qualidade estabelecidos pela classe de uso (CRUZ, 2001).

A outorga, por ser um instrumento que envolve direitos de uso, é um dos documentos exigidos no processo de licenciamento ambiental (Resolução CONAMA № 237/97). Portanto, os órgãos gestores de meio ambiente e de recursos hídricos deverão trabalhar de forma plenamente articulada, tendo em vista que a decisão a ser tomada é totalmente interdependente, ou seja: as eficiências de tratamento de efluentes definidas na licença de instalação ambiental $e$ as correspondentes cargas poluidoras remanescentes, deverão estar em perfeita consonância com as vazões que poderão ser alocadas para a diluição desses poluentes, e vice-versa (SILVA e MONTEIRO, 2004).

Pela complexidade da análise técnica dos impactos causados pelos diversos poluentes nos corpos receptores, bem como da respectiva análise da outorga, agora vista como instrumento de gestão integrada, é de todo recomendável que o processo de outorga de lançamento de efluentes se inicie de forma simples, mas eficiente. É prudente a adoção inicial de poucos parâmetros de qualidade da água nas análises de pedidos de outorga para lançamento de efluentes. 
A outorga de lançamento de efluentes, ao ser adotada para aqueles poluentes que representam os maiores impactos na bacia, deve estar integrada ao processo de enquadramento dos corpos de água, utilizando as mesmas prioridades de controle de poluição indicadas no processo de enquadramento, e estabelecendo, a partir daí, os parâmetros outorgáveis.

\section{TRANSPORTE DE MASSA}

Segundo Fisher 1979, a teoria do transporte de poluentes tem como base fundamental a combinação da lei de Fick com a Teoria da conservação das massas. Através desta combinação é possível fazer uma análise detalhada do comportamento de uma massa poluente em um campo de escoamento mono, bi ou tridimensional e também avaliar os três processos pertinentes ao movimento da massa poluente no corpo hídrico.

O primeiro processo, conhecido como difusão molecular, descreve o espalhamento da massa poluente causado pelo movimento molecular presente no sistema. O segundo trata da teoria advectiva que corresponde ao espalhamento da massa poluente através do movimento advectivo.

E finalmente, o terceiro que trata da difusão turbulenta. Este processo é controlado pelo coeficiente de difusão turbulenta que depende diretamente da energia de turbulência contida no campo de velocidade. Assim, quanto maior a turbulência, maior será o espalhamento (CHAGAS, 2005).

Deste modo, tomando como base um volume de controle, e fazendo a combinação das teorias citadas acima é possível chegar a equação geral da difusão advectiva, que leva em conta todos os processos combinados no transporte de massa.

$$
\frac{\partial C}{\partial t}+u \frac{\partial C}{\partial x}+v \frac{\partial C}{\partial y}+w \frac{\partial C}{\partial z}=D\left[\frac{\partial^{2} C}{\partial x^{2}}+\frac{\partial^{2} C}{\partial y^{2}}+\frac{\partial^{2} C}{\partial z^{2}}\right]
$$

Onde: 
$C$ : representa a concentração média em cada seção: $\left[M L^{-3}\right]$;

$U$ : representa a velocidade média em cada seção do rio: $\left[L T^{-1}\right]$;

$\mathrm{D}$ : representa o coeficiente de difusão turbulenta.

Considerando o caso de substâncias não conservativas e levando em conta a aplicação em apenas uma dimensão tem-se a seguinte equação:

$$
\frac{\partial C}{\partial t}+U \frac{\partial C}{\partial x}=\frac{\partial}{\partial x}\left(D_{x} \frac{\partial C}{\partial x}\right)-k C
$$

Onde $\mathrm{K}$ é o coeficiente de decaimento da substância $\left[\mathrm{T}^{-1}\right]$.

\section{TEORIA FUZZY}

Segundo Saavedra (2003) a lógica convencional trata as informações de modo binário, classificando-as como verdadeiras ou falsas. Talvez a definição desses dois estados da informação, em alguns casos, seja suficiente, porém, muitas experiências humanas necessitam de uma manipulação mais abrangente do que o simples tratamento de falso ou verdadeiro, sim ou não, certo ou errado.

Pode-se modelar, com a teoria fuzzy, diversas situações em que os dados envolvidos têm um certo 'grau' de incerteza ou imprecisão, ou a classificação de seus atributos não se resume em sim ou não, mas existe a possibilidade de: mais ou menos; talvez; um pouco mais; um pouco menos.

O fato de a teoria fuzzy dar esta flexibilidade de modelagem permite ao homem desenvolver algoritmos semelhantes ao pensamento humano. Um fator eminente dessa teoria é a sua capacidade de capturar conceitos intuitivos, além de considerar aspectos psicológicos utilizados pelos seres humanos em seu raciocínio usual, evitando que sua representação seja engessada por modelos tradicionais (OLIVEIRA, 1999).

Foi em 1965, pensando em atribuir significados a termos lingüísticos de cunho qualitativo, subjetivo, como 'perto', longe', alto', aproximadamente', que o matemático Lofti Zadeh, introduziu o conceito de conjuntos fuzzy (difusos). Através de tais conjuntos, seria possível armazenar dados não precisos em computadores, gerar respostas baseadas em 
informações vagas ou ambíguas, em processos análogos ao do raciocínio humano. Nesta lógica, são utilizados modelos matemáticos para mapear variáveis subjetivas, como frio, agradável e quente, para valores concretos que podem ser manipulados matematicamente. (SANTOS, 2008).

Aplicando a teoria fuzzy na equação 2 ela assume a seguinte forma:

$$
\frac{\partial \tilde{C}}{\partial t}+\tilde{U} \frac{\partial \tilde{C}}{\partial x}=\frac{\partial}{\partial x}\left(\tilde{D}_{x} \frac{\partial \tilde{C}}{\partial x}\right)-\tilde{k} \tilde{C}
$$

Onde: representa funções de pertinências para os parâmetros e para a variável de controle.

Considerando que a equação da difusão advectiva, na sua forma fuzzy não tem solução analítica simples, optou-se por um método numérico para sua solução. Assim, tendo em vista a sua simplicidade, o esquema numérico baseado no Método das Diferenças Finitas foi utilizado para resolver a equação de transporte. Para este trabalho utilizou-se o esquema semi-implícito de Crank-Nicolson, que garante estabilidade numérica no processo de solução. (ANDERSON et al., 1984).

Desta forma, discretizando a equação (3) seguindo um processo semi-implícito, a derivada parcial de $C$ com relação a $t$ é aproximada por:

$$
\tilde{A}(\alpha) \tilde{C}_{i-1}^{j+1}(\alpha)+\tilde{B}(\alpha) \tilde{C}_{i}^{j+1}(\alpha)+\tilde{D}(\alpha) \tilde{C}_{i+1}^{j+1}(\alpha)=\tilde{F}_{i}^{j}(\alpha)
$$

Onde: A, B, D são os coeficientes "fuzzys" da matriz [M], $\tilde{F}_{i}^{j}$ é o vetor com todas as informações conhecidas, e $\tilde{C}(\alpha)$ é o vetor solução do modelo para cada ponto do domínio e para cada tempo considerado.

De forma mais compacta tem-se:

$$
[\tilde{\varphi}(\alpha)][\tilde{C}(\alpha)]=[\tilde{F}(\alpha)]
$$

Onde: $\alpha$ é o nível de pertinência considerado.

\subsection{Cálculo do Risco Fuzzy e da Confiabilidade}


De acordo com Ganoulis (1994), se um evento, ou realização de um processo, é descrito por meio da lógica fuzzy, então a confiabilidade deste evento pode ser calculada como um número fuzzy. Considera-se que o sistema tem uma resistência $\tilde{R}$ e uma carga $\tilde{L}$, ambas representadas por números fuzzy. Uma medida de confiabilidade, ou uma margem de segurança que também caracteriza o desempenho do sistema, pode ser definida pela diferença entre a carga e a resistência. Esta diferença também é um número fuzzy, dado por:

$$
\tilde{M}=\tilde{R}-\tilde{L}
$$

Tem-se para cada função um intervalo de nível h:

$$
\tilde{M}(h)=\tilde{R}(h)-\tilde{L}(h)
$$

Onde:

$$
\begin{aligned}
& \tilde{R}(h)=\left[\tilde{R}_{1}(h), \tilde{R}_{2}(h)\right] \\
& \tilde{L}(h)=\left[\tilde{L}_{1}(h), \tilde{L}_{2}(h)\right]
\end{aligned}
$$

A medida marginal de segurança $\tilde{M}$ tem as possíveis condições:

Falha: $\tilde{M}(h)<0$

Confiabilidade: $\tilde{M}(h) \geq 0$

Segundo Ganoulis (1994), os índices fuzzy de confiabilidade e de falha são funcionais e dependem de várias funções como variáveis independentes que podem ser definidas como da seguinte forma:

> Índice de confiabilidade, ou garantia fuzzy:

$$
R_{c}=\frac{\int_{Z>0} \mu_{\tilde{M}}(m) d m}{\int_{Z} \mu_{\tilde{M}}(m) d m}
$$

Índice de falha, ou risco fuzzy: 


$$
R_{f}=\frac{\int_{Z<0} \mu_{\tilde{M}}(m) d m}{\int_{Z} \mu_{\tilde{M}}(m) d m}
$$

Onde:

$\mu_{\tilde{M}}$ : representa a função de pertinência;

$\mathrm{m}$ : representa um número real associado à função marginal de segurança.

A solução da equação matricial fuzzy (5) fornece os valores das concentrações em forma de funções de pertinência.

Os parâmetros "fuzzys" nesta equação são: a velocidade, o coeficiente de dispersão longitudinal e o coeficiente de decaimento. Neste caso, a fuzzificação destes parâmetros obedece à formulação proposta por CHAGAS (2005), conforme as equações abaixo:

$$
\begin{aligned}
& \mu_{k}=\frac{k-k_{L}}{k_{m}-k_{L}}, \text { para } k_{L} \leq k \leq k_{m} \\
& \mu_{k}=\frac{k-k_{u}}{k_{m}-k_{u}}, \text { para } k_{m} \leq k \leq k_{u}
\end{aligned}
$$

Onde: $k_{L}=0,75 k_{m} e k_{u}=1,25 k_{m}$

Com a solução do modelo proposto, pode-se determinar o risco de falha e a confiabilidade através das equações (10) e (11), tomando para a resistência os valores de concentrações definidos pela resolução CONAMA 357/2005.

\begin{tabular}{|c|c|c|c|c|c|c|c|c|c|c|c|c|c|}
\hline \multirow[b]{2}{*}{ Parâmetro } & \multicolumn{5}{|c|}{ Doces } & \multicolumn{4}{|c|}{ Salinas } & \multicolumn{4}{|c|}{ Salobras } \\
\hline & Esp. & 1 & 2 & 3 & 4 & Esp. & 1 & 2 & 3 & Esp. & 1 & 2 & $\overline{3}$ \\
\hline DBO & - & $<3$ & $<$ & $<10$ & - & - & - & - & - & - & - & - & - \\
\hline
\end{tabular}

Tabela 1: Parâmetros DBO e OD permissíveis segundo a Resolução CONAMA $357 / 2005$. 


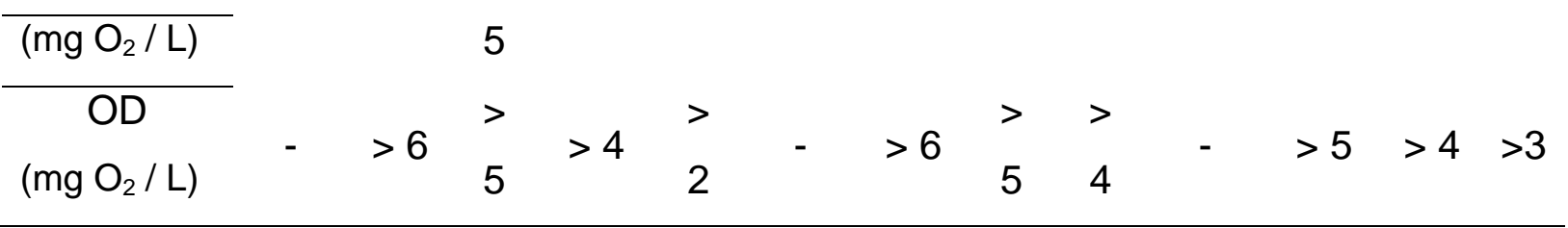

\section{ANÁLISE DOS RESULTADOS}

A seguir serão mostrados os resultados provenientes de simulações que consideraram lançamentos difusos, ao longo do canal. A taxa de lançamento foi definido como sendo uma grandeza fuzzy com valores $[0,000075 ; 0,0001 ; 0,000175]$ medidos em $\mathrm{mg} / \mathrm{m} / \mathrm{seg}$ (CHAGAS,2005).

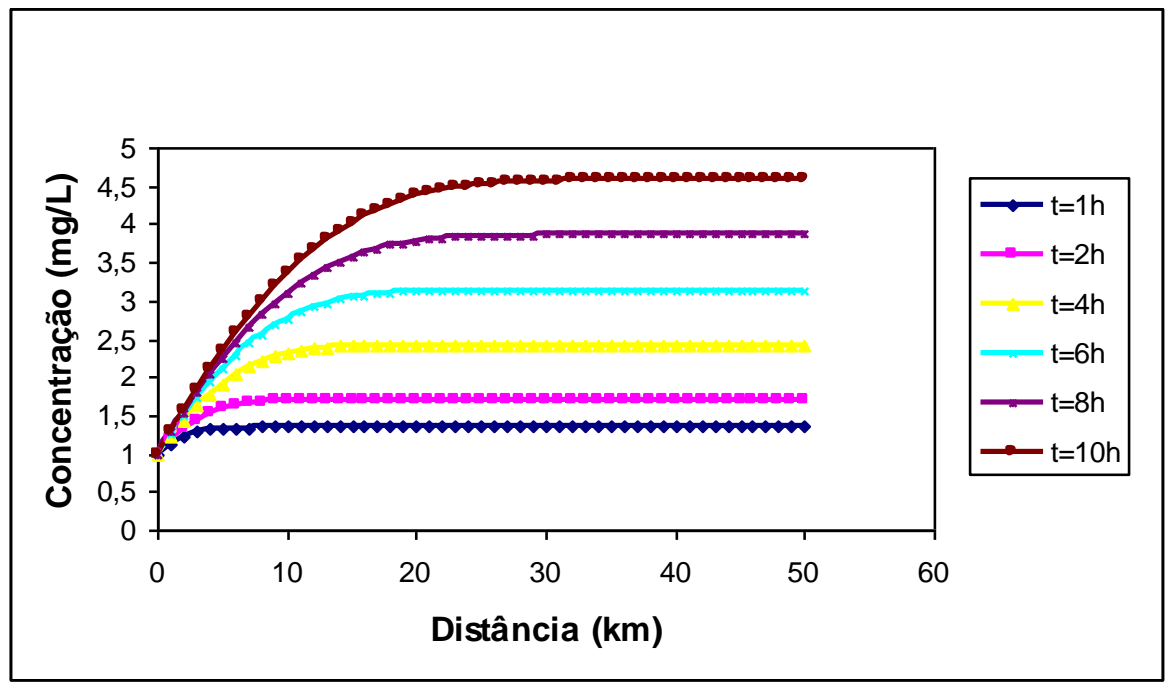

FIGURA 1 - Distribuição da concentração para um lançamento de uma carga difusa ao longo do canal para uma substância conservativa.

A Figura 1 mostra o perfil de concentração, para este tipo de lançamento, para diferentes tempos e para uma substância conservativa. Como podem ser observados, esses perfis são crescentes até atingir um ponto de estabilidade, onde o perfil se torna "quase horizontal". Na verdade, o perfil de cargas difusas só é horizontal quando a substância é não conservativa. Neste caso, o modelo se aproxima de uma ordenada igual à $S_{D} / K$, onde $S_{D}$ é a taxa de concentração difusa que é lançada, e $K$ é o coeficiente de 
decaimento da substância considerada. Uma análise mais criteriosa pode mostrar que, para este tipo de lançamento, as regiões mais críticas são as mais distantes da origem, onde o risco de falha do sistema é maior.

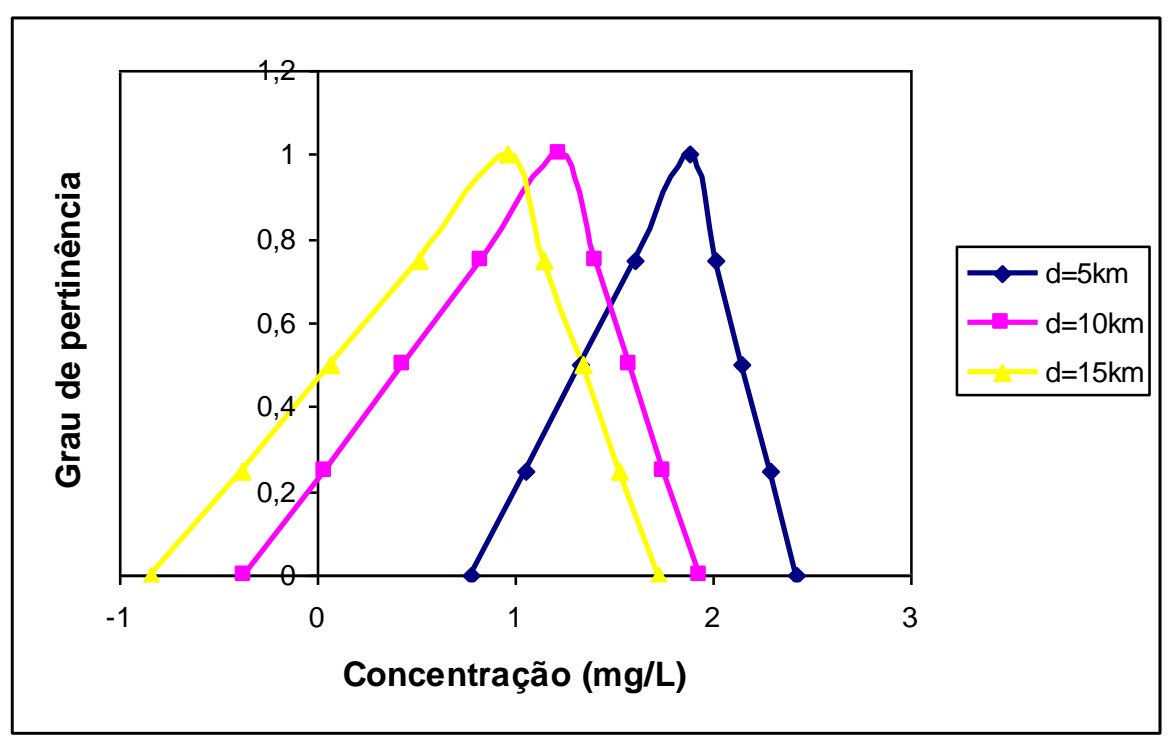

FIGURA 2 - Função marginal de segurança para diferentes seções em $\mathrm{t}=6 \mathrm{~h}$.

A Figura 2 comprova o que foi dito anteriormente com relação ao deslocamento das funções marginais de segurança, para maiores distancias da origem, para a esquerda. Como pode ser observado através da figura, para uma distancia de $15 \mathrm{~km}$, a função de pertinência se encontra bem mais a esquerda do que a marginal de segurança na seção 5 $\mathrm{km}$ da origem, para o mesmo tempo. Este deslocamento faz com que o risco de falha aumente. 


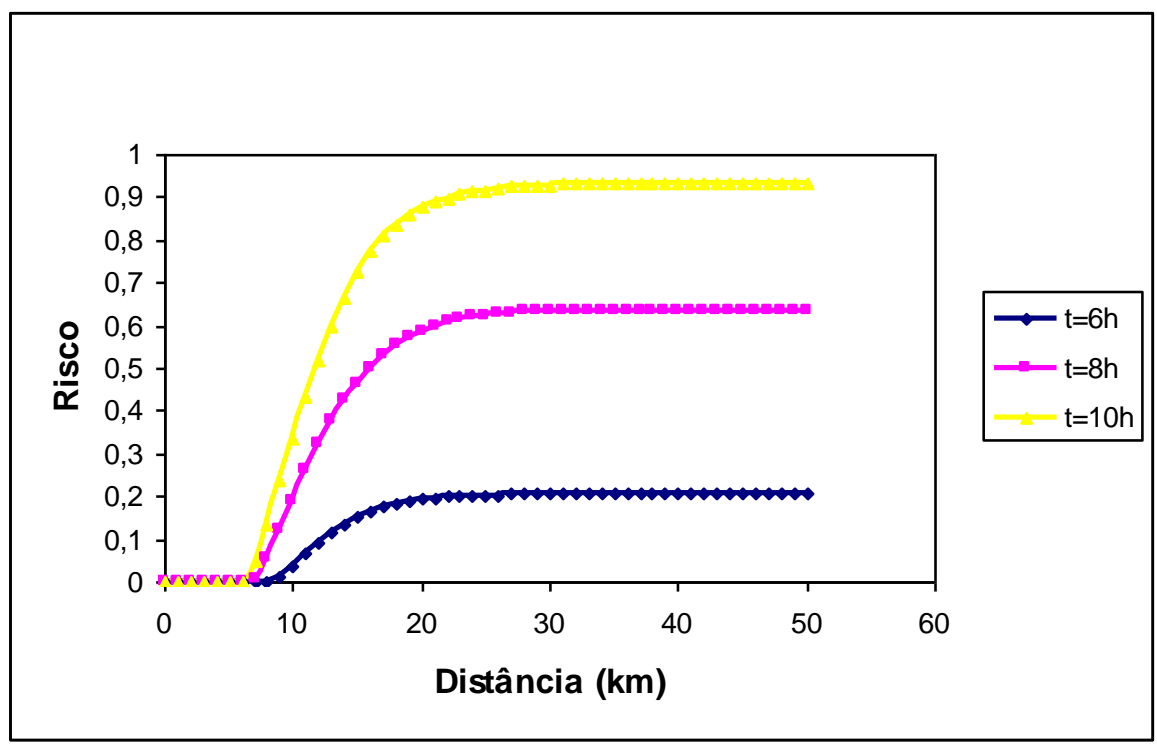

FIGURA 3 - Comportamento do risco com a distância em diferentes tempos.

A Figura 3 mostra os perfis do risco, em diferentes tempos, como função da distância. Este resultado comprova o que foi previsto na análise anterior. Como pode ser observado, a partir de $10 \mathrm{~km}$, o risco sofre um incremento positivo na medida em que se afasta da origem. Este resultado mostra a eficiência da metodologia proposta, onde uma combinação dos princípios de transporte de massa, juntamente com a associação da teoria fuzzy, permite que medidas de controle para concessão de outorga possa ser melhor avaliadas.

A Figura 4 compara as variações do risco e da garantia, como função do tempo, em uma seção a $10 \mathrm{~km}$ da origem. Este resultado mostra como esses funcionais se comportam em cada seção do canal, variando de acordo com o comportamento da nuvem poluente e suas concentrações. Desta forma, pode-se dizer que o risco e a garantia são dependentes diretos do comportamento do campo de concentração que, por sua vez, representa uma resposta do corpo hídrico e sua capacidade de diluição, a um determinado lançamento. 


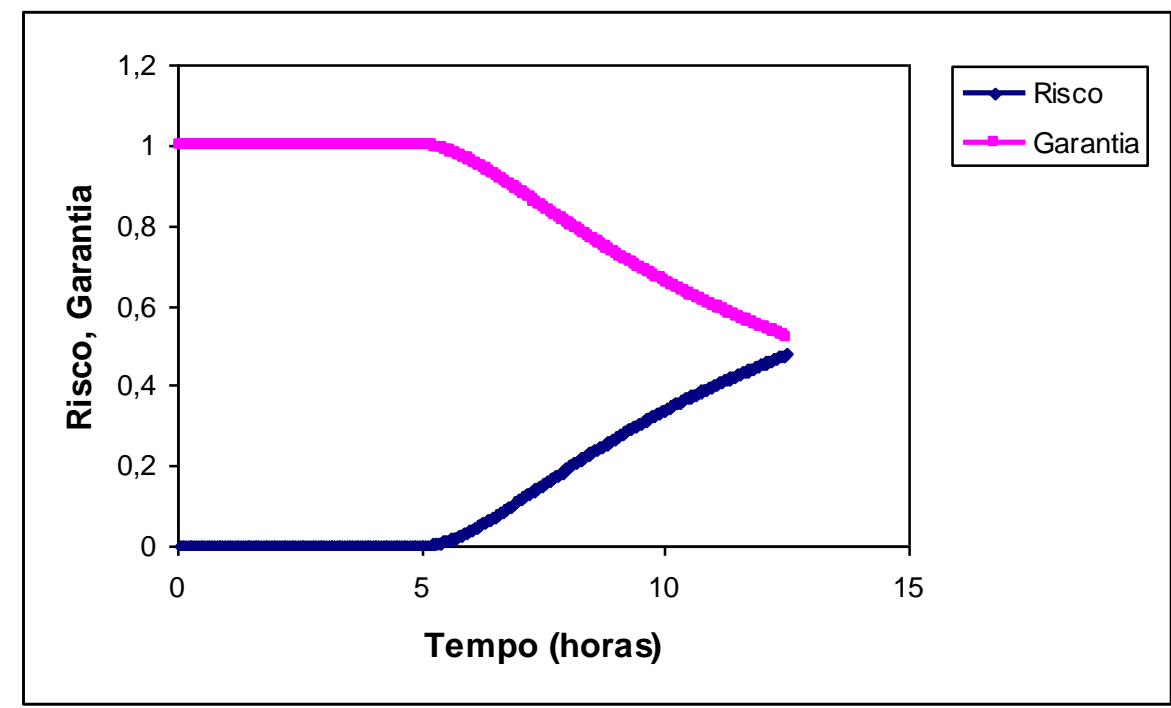

FIGURA 4 - Comportamento do risco e da garantia ao longo do tempo para um lançamento de uma carga difusa ao longo do canal para uma substância conservativa.

\section{CONCLUSÕES}

Após a aplicação do modelo proposto em um rio natural sujeito a lançamentos difusos de efluentes, uma análise foi realizada com vistas à análise de concessão de outorga de lançamentos. A aplicação da formulação fuzzy na Equação de Balanço de Massa mostrou-se eficiente no cálculo do risco e da garantia, os quais representam medidas de controle para o sistema hídrico em questão.

O uso da Teoria Fuzzy em Modelos de Balanço de Massa, que possibilita transformar esses modelos em Equações Diferenciais Fuzzys, quando tratado adequadamente permite que se obtenham Funções de Pertinências para as variáveis de controle. No caso do estudo em questão, a variável de controle é a concentração do poluente que é lançado em um corpo hídrico. Desta forma, a metodologia proposta é possível determinar campos de concentração, em sua forma fuzzy, para que se desenvolvam métodos de cálculo dos campos de risco e de garantia, em toda a extensão do corpo hídrico. Os resultados mostraram que o risco de falha para um determinado rio, que recebe lançamentos de efluentes, proveniente de concessões de outorga, depende do tipo de lançamento, bem como da concentração dos efluentes que estão sendo lançados. 
Para lançamentos difusos contínuos, as análises mostraram que o risco cresce com o tempo até atingir o valor desta função definido através da concentração de diluição. Assim, o risco tende a aumentar, em toda a extensão do canal, até se estabilizar no valor estabelecido no ponto de lançamento. Este valor só vem a cair se a vazão do rio aumentar. Neste caso, à medida que a vazão aumenta, o risco diminui. Isto mostra a importância de se ter um conhecimento prévio da capacidade hidrológica das bacias as quais esses rios pertencem.

\section{BIBLIOGRAFIA}

ANDERSON, D. A.; TANNEHILL, J. C.; PLETCHER, R. H. Computational Fluid Mechanics and Heat Transfer. Hemisphere Publishing Corporation, 1984.

AZEVEDO, L. G. T. et al. Sistemas de Suporte à Decisão para a Outorga de Direitos de Uso da Água no Brasil. In: AZEVEDO, L. G. T; MEJIA, A (Coord). Série Água Brasil. Brasília: Banco Mundial, 2003. 48p.

CHAGAS (2005). Perspectivas da Aplicação da Teoria Fuzzy para o cálculo de risco em sistemas hidrodinâmicos. Tese defendida no Departamento de Engenharia Hidráulica e Ambiental da Universidade Federal do Ceará como parte dos requisitos para obtenção do título de doutor em recursos hídricos.

CRUZ, J. C. - Disponibilidade hídrica para outorga: avaliação de aspectos técnicos e conceituais. Porto Alegre, 2001. 189 p. Tese (Doutorado em Engenharia de Recursos Hídricos e Saneamento Ambiental). Departamento de Hidráulica e Saneamento. Universidade Federal do Rio Grande do Sul.

NAHON, I. M (2006). Sistema de Apoio a Análise de Outorga de Lançamento de Efluentes para a Variável Demanda Bioquímica de Oxigênio: Estudo de caso da Bacia do Alto Iguaçu. Dissertação apresentada como requisito parcial a obtenção do título de mestre em Engenharia de Recursos Hídricos e Ambiental, Universidade Federal do Paraná.

OLIVEIRA JR., H. A., Lógica Difusa: Aspectos Práticos e Aplicações, Interciência, 1999.

ROQUES, T. V. P. (2006). "Aplicação de modelos computacionais na análise de outorga para diluição de efluentes em corpos de água - fontes pontuais e difusas". Dissertação de Mestrado. Programa de Pós Graduação em Engenharia Ambiental da Universidade Federal do Espírito Santo. Vitória, 2006. 
SAAVEDRA, O. R., Introdução aos Conjuntos Difusos - Notas de aula - Inteligência Artificial, Universidade Federal do Maranhão, 2003.

SANTOS, S. H. L. Aplicação da Teoria dos Números Difusos em um Modelo de Operação de Reservatório, para estudar o Comportamento Vazão Regularizada e do Rendimento., Dissertação de Mestrado em Engenharia Civil, Universidade Federal do Ceará, Fortaleza, 2008.

SILVA, L. M. C.; MONTEIRO, R. A. Outorga de direito de uso de recursos hídricos: uma das possíveis abordagens. IN: MACHADO, C. J. S. (Org.). Gestão de águas doces. Rio de Janeiro: Interciência. 2004. cap. 5, p. 135-178.

\section{AGRADECIMENTOS}

Os autores agradecem ao CNPQ e a CAPES, pelo apoio à pesquisa. 